\title{
Relación entre Apoyo Social percibido, Severidad de Insomnio y Somnolencia Diurna en Adultos Mayores
}

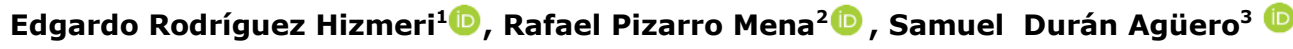 \\ ${ }^{1}$ Universidad San Sebastián, Programa de Magíster en Kinesiología Gerontológica y Geriátrica. Chile \\ ${ }^{2}$ Universidad San Sebastián, Facultad de Salud, Escuela de Kinesiología. Chile \\ ${ }^{3}$ Universidad San Sebastián, Facultad de Ciencias Para el Cuidado de la Salud, Escuela de Nutrición y \\ Dietética. Chile
}

Cómo referenciar este artículo/

How to reference this article:
Rodríguez Hizmeri E, Pizarro Mena R, Durán Agüero S. Relación entre Apoyo Social percibido, Severidad de Insomnio y Somnolencia Diurna en Adultos Mayores.Mem. Inst. Investig. Cienc. Salud. 2020; 18(3): 34-42

\section{RE S U M E N}

El apoyo social es un importante factor protector de la salud de los adultos mayores (AM). Asimismo, un buen dormir es fundamental para su bienestar y funcionalidad, siendo las alteraciones del sueño un importante problema de salud pública en este grupo etario. El objetivo del estudio fue determinar la relación entre apoyo social percibido, insomnio y somnolencia diurna en AM residentes en Chillán, Chile. Se realizó un estudio cuantitativo, no experimental, correlacional, de corte transversal. Se aplicó la versión chilena de la Escala de Apoyo Social Percibido (MOS), el Índice de severidad del insomnio (ISI), y la Escala de somnolencia diurna de Epworth a AM pertenecientes a agrupaciones comunitarias. Se entrevistaron a 202 AM de ambos sexos, de edad $72,3 \pm 6,2$ años. Se presentaron correlaciones positivas entre escolaridad y severidad del insomnio $(p<0,05)$ y entre severidad del insomnio y somnolencia $(p<0,001)$, además, se encontraron correlaciones negativas entre severidad del insomnio y apoyo social percibido, tanto en forma global como por dimensiones $(p<0,05)$. La dimensión "Interacción social positiva" se correlacionó también de forma negativa con la somnolencia diurna. Los AM participantes del estudio que presentaron un alto nivel de apoyo social presentaron una menor prevalencia y severidad del insomnio.

Palabras clave: Adulto mayor, apoyo social, sueño, insomnio, somnolencia diurna.

\section{Relationship between perceived Social Support, Severity of Insomnia and Daytime Sleepiness in Elderly}

\begin{abstract}
A B S T R A C T
Social support is an important protective factor for the health of elderly (E).Likewise, good sleep is fundamental for their well-being and functionality, being sleep disorder an important public health problem in this age group. The objective of this study was to determine the relationship between perceived social support, insomnia and daytime sleepiness in elderly residents of Chillán, Chile. A quantitative, non-experimental, correlational, cross-sectional study was carried out. The Chilean version of the Perceived Social Support Scale (MOS), the Index of severity of insomnia (ISI) and the Daytime Sleepiness Scale of Epworth was applied to elderly belonging to community groups. Two hundred two elderly of both sexes were interviewed, age $72.3 \pm 6.2$ years. There were positive correlations between schooling and severity of insomnia ( $p$


$<0.05)$ and between severity of insomnia and somnolence $(p<0.001)$. In addition, there were negative correlations between severity of insomnia and perceived social support, both globally and by dimensions $(p<0.05)$. The dimension "Positive social interaction" also correlates negatively with daytime sleepiness. The elderly participants of the study who presented a high level of social support had a lower prevalence and severity of insomnia.

Keywords: Elderly, social support, sleep, insomnia, daytime sleepiness.

\section{INTRODUCCIÓN}

Durante las últimas décadas, Chile ha experimentado importantes cambios demográficos, epidemiológicos y sociales. Entre ellos, el aumento de la esperanza de vida y la disminución de la mortalidad han derivado en un envejecimiento progresivo de la población, sin embargo, este aumento en la cantidad de años vivida no siempre se acompaña de vivir estos años con una buena calidad de vida ${ }^{(1)}$.

El envejecimiento está caracterizado por la disminución progresiva de la capacidad funcional en todos los tejidos y órganos del cuerpo, además de conllevar una mayor carga de problemas de salud asociados a estilos de vida, lo que plantea como desafío la conservación de la calidad de vida y la autonomía de los $\mathrm{AM}^{(1-3)}$. Para tener una buena calidad de vida es fundamental presentar un descanso adecuado. Una de las alteraciones de salud más frecuentes entre los AM son los trastornos de sueño que alcanza a un $51,8 \%$ en población AM chilena ${ }^{(4)}$, relacionada tanto con procesos fisiológicos ligados al envejecimiento, como a factores externos, entre los cuales existen procesos que forman parte de una red compleja de mecanismos psicosociales y condicionantes socioestructurales, a través de diversas vías: conductas en salud (higiene del sueño, uso de fármacos, dieta, ejercicio, etc.), factores psicológicos (autoeficacia, autoestima, bienestar, depresión, ansiedad) y factores psicosociales: redes sociales y apoyo social ${ }^{(1,5)}$. Diversos estudios muestran relación entre una mala calidad o alteraciones del sueño con una menor calidad de vida ${ }^{(6)}$, salud en general ${ }^{(7)}$, bienestar psicológico ${ }^{(8)}$, y diversos factores cardiovasculares ${ }^{(1,9)}$.

En el AM, las alteraciones del sueño y los desórdenes neurodegenerativos se han convertido en un problema mayor de salud pública, asociándose con una mayor fragilidad, morbilidad y mortalidad. Diversos estudios sostienen que las interrupciones en el sueño nocturno, así como las alteraciones en su duración (es decir, sueño demasiado breve o muy largo) son predictores de deterioro cognitivo ${ }^{(10)}$. Como se mencionaba anteriormente, la calidad del sueño y sus alteraciones se relacionan con una serie de factores, entre los cuales se cuenta el apoyo social, que puede ser conceptualizado a partir de dos perspectivas, una cuantitativa-estructural y otra cualitativa-funcional.La primera evalúa la cantidad de vínculos o relaciones que establece el individuo con su red social, que correspondería al apoyo social recibido; y la segunda se centra en la existencia de relaciones significativas y la evaluación o apreciación subjetiva que realiza el individuo de la adecuación del apoyo que recibe, la que correspondería al apoyo social percibido ${ }^{(11)}$.

La relación entre apoyo social percibido y alteraciones del sueño, especialmente en AM es un área poco explorada, sobre la cual no se ha encontrado al momento de la presente investigación estudios desarrollados en Chile, si bien en otros países se reporta relación del apoyo social con alteraciones del sueño en población adulta ${ }^{(12)}$, problemas de sueño en adultos mayores ${ }^{(13)}$, calidad de sueño en pacientes con demencia ${ }^{(14)}$, y -en conjunto con vulnerabilidad y estilos de afrontamiento - sobre la incidencia y persistencia del insomnio ${ }^{(15)}$.

Por lo tanto, el propósito del presente estudio es determinar la relación entre apoyo social percibido, insomnio y somnolencia diurna en AM de la ciudad de Chillán.

\section{MATERIAL Y MÉTODOS}

Estudio analítico transversal. Se realizó un muestreo aleatorio por conveniencia, a partir de una población de 30.972 AM residentes en la ciudad de Chillán (Capital de la Región del Ñuble), y según proyecciones para el 2018 elaboradas por el Instituto Nacional de Estadísticas (INE), se calculó el tamaño muestral mediante la siguiente 
fórmula, considerando un nivel de confianza ( $Z$ ) del $90 \%$, proporciones $\mathrm{p}$ y q de $0,5 \mathrm{y}$ un error muestral (e) de $6 \%$, se obtuvo un tamaño muestral $n$ de 202 AM. Para reclutar a los participantes, se acudió a lugares de encuentro de AM, tales como Casa del AM, juntas de vecinos, clubes de AM, actividades comunitarias, entre otros; para lo cual se contó previamente con una amplia base de datos de contacto. Fueron incluidos los AM de 60 años y más, residentes en la ciudad, que manifestaron su voluntad de participar en el estudio a través de la firma de un Consentimiento Informado, se excluyeron quienes presentaron alteraciones psiquiátricas, neurológicas, sensoriales o de otra índole que podrían afectar su nivel de discernimiento o la comprensión de los cuestionarios aplicados y completar en su totalidad los instrumentos aplicados. Además, AM que estuviesen sometidos a algún tratamiento farmacológico que podía alterar la calidad de sueño, que padecían, al momento de la aplicación de los instrumentos, alguna enfermedad aguda que afectaba directamente su calidad de sueño (ej. infección respiratoria aguda), con diagnóstico de apnea obstructiva del sueño.

\section{Instrumentos de evaluación:}

\section{Índice de Severidad del Insomnio (ISI)}

Se aplicó el Índice de Severidad del Insomnio, cuestionario breve, sencillo y autoadministrado que consta de siete ítems: los tres primeros evalúan la gravedad del insomnio con puntuaciones que van de 0 a 4 ( 0 =nunca, $1=$ leve, $2=$ moderado, $3=$ grave y $4=$ muy grave), y los cuatro restantes miden la satisfacción del sueño (desde 0 muy satisfecho a 4 muy insatisfecho), las interferencias del sueño con el funcionamiento diario, la percepción del problema del sueño por parte de los demás y el nivel de preocupación del paciente, estas 3 últimas preguntas con puntuaciones que van de 0 a $4(0=\text { nada, } 1=\text { un poco, } 2=\text { =algo, } 3=\text { mucho y } 4=\text { muchísimo })^{(16)}$.

Escala de somnolencia diurna de Epworth

Se aplicó la Escala de somnolencia diurna de Epworth, cuestionario autoadministrado que evalúa la propensión a quedarse dormido en ocho situaciones sedentarias diferentes. Consta de 8 ítems con puntuaciones posibles de 0 a 3 ( $0=$ nunca, $1=$ leve, $2=$ moderado y $3=$ severo). Al final se puntúa entre $0-24$, donde puntajes altos representan mayor grado de somnolencia, considerándose somnoliento $>10$ y somnolencia excesiva $>15$ puntos ${ }^{(17,18)}$.

\section{Escala de apoyo social percibido (MOS)}

Se aplicó la versión chilena, elaborada por Poblete et. al. ${ }^{(1)}$, de la escala de apoyo social percibido MOS, desarrollada a partir del estudio Medical Outcomes Study Social Support Survey (MOS-SSS). Esta escala consta de 19 ítems tipo Likert en escala de 1 a 5 puntos cada uno ( $1=$ nunca, $2=$ pocas veces, $3=$ algunas veces, $4=$ la mayoría de las veces, y $5=$ siempre), donde una mayor puntuación indica un mayor apoyo social percibido. El apoyo social percibido es tratado como una variable binaria, con categorías de apoyo social "bajo" o "alto". sí, los participantes que reportasen recibir apoyo "la mayoría de las veces" o "siempre" se considera que reciben un alto nivel de apoyo social percibido, mientras aquellos que respondan que esto ocurre "nunca", "pocas veces" o "algunas veces" son agrupados como personas que reciben un bajo nivel de apoyo social percibido. Los ítems de la escala están agrupados en cuatro factores, Interacción social positiva: ítems 7, 9, 11, 14, 16, 18 y 19; Apoyo afectivo: ítems 6, 10 y 20; Apoyo instrumental: ítems 2, 5, 12 y 15; Apoyo emocional informativo: ítems 3, 4, 8 y 13. La escala MOS se encuentra ampliamente validada en la literatura, y ha demostrado excelentes características psicométricas, que se mantienen en la versión modificada por Poblete. Además, se les consultó por antecedentes sociodemográficos (escolaridad, salud, ocupación, etc).

La investigación fue aprobada por Comité Ético del Servicio de Salud Ñuble. Se entregó a cada AM participante un documento de Consentimiento Informado. Este estudio cumple los estándares éticos del Consejo de Organizaciones Internacionales de las Ciencias Médicas (CIOMS) y de la declaración de Helsinki de 1975, revisada en el 2000. 


\section{Estadística}

Los datos fueron procesados mediante el programa estadístico SPSS, versión 22.0. Se consideró significativo un $\mathrm{p}$-valor $<0,05$. Las variables cualitativas fueron descritas mediante frecuencias y porcentajes. Las variables continuas fueron descritas utilizando media, mediana, moda y desviación estándar. Las correlaciones entre variables numéricas se analizaron mediante la pruebas $\mathrm{R}$ de Pearson; para la comparación entre grupos se utilizó la prueba $t$ de Student para muestras independientes.

\section{RESULTADOS}

Se entrevistaron a 202 AM de ambos sexos, con una media de $72,3 \pm 6,2$ años. En cuanto al sexo, se observó un claro predominio femenino $(82,6 \%)$. En cuanto al nivel educacional, el predominante fue la Secundaria (incompleta-completa), con un $36,6 \%$ del total. Un $84,1 \%$ de los participantes estaba pensionado o jubilado, siendo éste también el segmento predominante entre los hombres, de quienes representa un $91,43 \%$. El $45,5 \%$ afirmó que la calidad de su dormir era "buena", seguida por "bastante buena" $(26,2 \%)$; sólo un $5,9 \%$ sostuvo tener una "muy mala" calidad de sueño. Un $41,5 \%$ de la muestra presentó insomnio, siendo el insomnio subclínico el grado de severidad predominante $(29,2 \%)$; sólo un $4,4 \%$ de los participantes presentó insomnio clínico grave, lo cual correspondió a 9 participantes. El 2,9\% de los participantes presentó somnolencia diurna (Tabla 1).

Tabla 1. Características generales de la muestra $(n=202)$

\begin{tabular}{|c|c|c|c|c|}
\hline & Media & DE & Mínima & Máximo \\
\hline Edad (años) & 72,3 & 6,3 & 60 & 91 \\
\hline Educación & Sin educación & $\begin{array}{l}\text { Primaria } \\
\text { (incompleta- } \\
\text { completa) }\end{array}$ & $\begin{array}{l}\text { Secundaria } \\
\text { (incompleta- } \\
\text { completa) }\end{array}$ & Superior \\
\hline Número (\%) & $5(2,5)$ & $69(34,2)$ & $74(36,6)$ & $54(26,7)$ \\
\hline Ocupación & Trabajador & $\begin{array}{l}\text { Pensionado o } \\
\text { jubilado }\end{array}$ & Dueño/a de casa & Cesante \\
\hline Número (\%) & $3(1,5)$ & $170(84,1)$ & $27(13,4)$ & $2(1,0)$ \\
\hline Salud & $\begin{array}{l}\text { Fonasa } \\
193(96,5)\end{array}$ & $\begin{array}{l}\text { Isapre } \\
7(3,4)\end{array}$ & $\begin{array}{l}\text { Dipreca/capredena } \\
1(0,5)\end{array}$ & $\begin{array}{l}\text { Prais } \\
1(0,5)\end{array}$ \\
\hline Enfermedades & $\begin{array}{l}\text { HTA } \\
63,8\end{array}$ & $\begin{array}{l}\text { DM } 2 \\
22,3\end{array}$ & $\begin{array}{l}\text { Artrosis y otras } \\
30,7\end{array}$ & $\begin{array}{l}\text { Otras } \\
18,3\end{array}$ \\
\hline $\begin{array}{l}\text { Calidad subjetiva } \\
\text { del sueño }\end{array}$ & Bastante buena & Buena & Mala & Bastante mala \\
\hline & $53(26,2)$ & $92(45,5)$ & $12(5,9)$ & $45(22,4)$ \\
\hline $\begin{array}{l}\text { Indica de severidad } \\
\text { de Isomnio }\end{array}$ & Sin insomnio & $\begin{array}{l}\text { Insomnio } \\
\text { subclínico }\end{array}$ & $\begin{array}{l}\text { Insomnio clínico } \\
\text { moderado }\end{array}$ & $\begin{array}{l}\text { Insomnio clínico } \\
\text { grave }\end{array}$ \\
\hline Escala de Apoyo & Apoyo Alto & Aроуо Bajo & & \\
\hline & $133(65,8)$ & $69(34,2)$ & & \\
\hline
\end{tabular}

Con respecto al apoyo social percibido, la mitad de los participantes obtuvo una puntuación mayor a 82,5 puntos, y la media correspondió a 76,4 puntos, lo que indicó un alto nivel de apoyo social percibido por AM. Se observó, además, que la dimensión mejor evaluada fue Apoyo afectivo, mientras que la más baja correspondió a Apoyo instrumental. Al interpretar las puntuaciones totales de la escala, se obtuvo que un $65,8 \%$ refirió un alto nivel de apoyo social percibido (Tabla 2 ). 
Tabla 2. Medidas de resumen y consistencia interna de las dimensiones de Apoyo Social Percibido (MOS)

\begin{tabular}{lllllll}
\hline Dimensiones MOS & Media & $\begin{array}{l}\text { Media } \\
\text { ponderada }\end{array}$ & DE & Mínimo & Mediana & Máximo \\
\hline Interacción social positiva & 29,67 & 4,24 & 6,88 & 7 & 33 & 35 \\
Apoyo afectivo & 13,59 & 4,53 & 2,58 & 4 & 15 & 15 \\
Apoyo instrumental & 16,46 & 4,12 & 4,59 & 4 & 18,5 & 20 \\
Apoyo emocional - informativo & 16,7 & 4,18 & 4,01 & 4 & 18 & 20 \\
MOS total & 76,42 & 10,92 & 16,34 & 26 & 82,5 & 90 \\
\hline
\end{tabular}

La Tabla 3 compara los resultados obtenidos por hombres y mujeres en las escalas Epworth, ISI y MOS. No se observa una tendencia clara a una mayor puntuación entre hombres o mujeres con respecto a estas escalas; asimismo, al comparar las medias de ambos grupos, no se obtuvo diferencias estadísticamente significativas. Con respecto a la presencia de enfermedades crónicas, las personas portadoras de enfermedades crónicas tenían una mayor prevalencia y severidad de insomnio, más somnolencia diurna, y un menor apoyo social percibido, sin embargo, sin ser estas diferencias estadísticamente significativas (datos no mostrados).

Tabla 3. Comparación entre escalas Epworth, ISI y MOS según sexo en adultos mayores

\begin{tabular}{|c|c|c|c|c|}
\hline \multicolumn{2}{|c|}{, } & Hombre $(n=35)$ & Mujer $(n=167)$ & Valor $\mathbf{p}$ \\
\hline \multicolumn{2}{|c|}{$\begin{array}{l}\text { Índice de severidad del Insomnio } \\
\text { (puntaje) }\end{array}$} & $6,2 \pm 5,8$ & $6,9 \pm 6,6$ & 0,54 \\
\hline \multicolumn{2}{|c|}{$\begin{array}{l}\text { Escala de Somnolencia diurna de Epworth } \\
\text { (puntaje) }\end{array}$} & $4,7 \pm 4,1$ & $4,0 \pm 3,9$ & 0,37 \\
\hline Escala de & Interacción positiva & $28,7 \pm 8,9$ & $29,9 \pm 6,4$ & 0,47 \\
\hline Apoyo Social & Apoyo afectivo & $13,0 \pm 3,4$ & $13,7 \pm 2,4$ & 0,24 \\
\hline \multirow[t]{3}{*}{ Percibido } & Apoyo instrumental & $16,5 \pm 5,6$ & $16,5 \pm 4,4$ & 0,94 \\
\hline & $\begin{array}{l}\text { Apoyo emocional- } \\
\text { informativo }\end{array}$ & $16,5 \pm 5,1$ & $16,8 \pm 3,4$ & 0,75 \\
\hline & MOS total & $74,7 \pm 21,8$ & $76,8 \pm 15,0$ & 0,49 \\
\hline
\end{tabular}

Valores expresados en media \pm DE. Prueba T de Student

La Tabla 4 muestra la correlación entre las variables cuantitativas observadas en el estudio, se observan correlaciones positivas, débiles y estadísticamente significativas entre: escolaridad y severidad del insomnio $(p<0,05)$, y entre severidad del insomnio y somnolencia $(p=0,001)$.Además, existen correlaciones negativas, débiles, pero estadísticamente muy significativas, entre severidad del insomnio y apoyo social percibido, tanto en forma global como por dimensiones $(p<0,05)$. La dimensión "Interacción social positiva" se correlaciona también de forma negativa, débil y estadísticamente significativa con la somnolencia diurna.

Tabla 4. Correlación de Pearson entre MOS, edad, somnolencia diurna e insomnio

\begin{tabular}{|c|c|c|c|c|c|c|c|c|c|c|}
\hline & & \multirow[b]{2}{*}{ Edad } & \multirow[b]{2}{*}{ Estudios } & \multirow[b]{2}{*}{ Epworth } & \multirow[b]{2}{*}{ ISI } & \multirow[b]{2}{*}{$\begin{array}{c}\text { Interacción } \\
\text { social } \\
\text { positiva }\end{array}$} & \multicolumn{2}{|r|}{ MOS } & \multirow[b]{2}{*}{$\begin{array}{c}\text { Apoyo } \\
\text { emocional } \\
\text { informativo }\end{array}$} & \multirow[b]{2}{*}{ Total } \\
\hline & & & & & & & $\begin{array}{l}\text { Apoyo } \\
\text { afectivo }\end{array}$ & $\begin{array}{c}\text { Apoyo } \\
\text { instrumental }\end{array}$ & & \\
\hline $\begin{array}{l}\text { Edad } \\
\text { Escolaridad }\end{array}$ & $\begin{array}{l}r \\
r\end{array}$ & $-0,073$ & $\begin{array}{l}-0,073 \\
1\end{array}$ & $\begin{array}{l}-0,017 \\
0,093\end{array}$ & $\begin{array}{l}-0,016 \\
-0,164 *\end{array}$ & $\begin{array}{r}-0,068 \\
0,115\end{array}$ & $\begin{array}{l}-0,021 \\
0,005\end{array}$ & $\begin{array}{l}-0,038 \\
0,065\end{array}$ & $\begin{array}{l}-0,069 \\
0,158^{*}\end{array}$ & $\begin{array}{l}-0,06 \\
0,106\end{array}$ \\
\hline $\begin{array}{l}\text { Epworth } \\
\text { ISI }\end{array}$ & $\begin{array}{l}r \\
r\end{array}$ & $\begin{array}{l}-0,017 \\
-0,016\end{array}$ & $\begin{array}{l}0,093 \\
-0,164 *\end{array}$ & $\begin{array}{l}1 \\
0,222 * *\end{array}$ & $\begin{array}{l}0,222 * * \\
1\end{array}$ & $\begin{array}{r}-0,14 \\
-0,361^{* *}\end{array}$ & $\begin{array}{l}-0,061 \\
-0,209 * *\end{array}$ & $\begin{array}{l}-0,133 \\
-0,332 * *\end{array}$ & $\begin{array}{l}-0,061 \\
-0,3 * *\end{array}$ & $\begin{array}{l}-0,121 \\
-0,352 * *\end{array}$ \\
\hline $\begin{array}{l}\text { Inter. } \\
\text { social } \\
\text { positiva }\end{array}$ & $r$ & $-0,068$ & 0,115 & $-0,14 *$ & $-0,361 * *$ & 1 & $0,754 * *$ & $0,737 * *$ & $0,837 * *$ & $0,953 * *$ \\
\hline $\begin{array}{l}\text { Apoyo } \\
\text { afectivo }\end{array}$ & $r$ & $-0,021$ & 0,005 & $-0,061$ & $-0,209$ & $0,754 * *$ & 1 & $0,654 * *$ & $0,692 * *$ & $0,829 * *$ \\
\hline $\begin{array}{l}\text { Apoyo } \\
\text { instrumental }\end{array}$ & $r$ & $-0,038$ & 0,065 & $-0,133$ & $-0,332 * *$ & $0,737 * *$ & $0,654 * *$ & 1 & $0,721 * *$ & $0,872 * *$ \\
\hline $\begin{array}{l}\text { A. } \\
\text { emocional- } \\
\text { infor }\end{array}$ & $r$ & $-0,069$ & 0,158 & $-0,061$ & $-0,3 *$ & $0,837 * *$ & $0,692 * *$ & $0,721 * *$ & 1 & $0,91 * *$ \\
\hline $\begin{array}{l}\text { MOS - } \\
\text { Total }\end{array}$ & $r$ & $-0,06$ & 0,106 & $-0,121$ & $-0,352 * *$ & $0,953 * *$ & $0,829 * *$ & $0,872 * *$ & $0,91 * *$ & 1 \\
\hline
\end{tabular}




\section{DISCUSIÓN}

El principal resultado del estudio fue que los $A M$ participantes del estudio que presentaron un alto nivel de apoyo social presentaron una menor prevalencia y severidad del insomnio.

Respecto a los antecedentes sociodemográficos la muestra observada presenta un notorio predominio femenino, esto podría explicarse en parte dada la mayor esperanza de vida de las mujeres, y por ende el mayor porcentaje femenino en la población AM chilena ${ }^{(19)}$. Con respecto a otros estudios sobre sueño, coincide con lo reportado por Viana et al. ${ }^{(13)}$, cuya muestra está compuesta por un $68,5 \%$ de mujeres. Además, diversos estudios indican una mayor participación femenina en organizaciones comunitarias, en especial en los segmentos de mayor edad, lo cual podría explicar el predominio femenino entre los participantes de la presente investigación, todos asistentes a agrupaciones de $\mathrm{AM}^{(20)}$.

En cuanto a las características del sueño de las personas encuestadas, la mayoría refirió una valoración positiva (buena o bastante buena) de su calidad de sueño. Esto es discordante con lo reportado por Eshkooret al. ${ }^{(14)}$ quienes reportan un $41 \%$ de AM con alteraciones del sueño, si bien su investigación abarca a pacientes con deterioro cognitivo o demencia, a diferencia de la población observada en este estudio, constituida por personas en general autovalentes, participantes activos de talleres comunitarios; este hecho da un primer atisbo que la participación social activa puede ser un elemento favorable para la calidad de sueño.

La prevalencia de insomnio entre los participantes corresponde a un 41,5\%; en cuanto a su severidad, predomina en su forma subclínica (29,2\%). Este porcentaje es levemente mayor al observado en el estudio MAPT-Sleep realizado en Francia por Gabelleet al. ${ }^{(21)}$ que muestra una prevalencia de insomnio de $21,2 \%$ en pacientes AM sin deterioro cognitivo.

Los resultados de la Escala de Somnolencia de Epworth muestran que la mayor parte de los participantes no presentaba esta alteración, es decir, la mayoría de estos AM no tiene dificultad para mantenerse alerta y despierto durante el día. Esto difiere con el $27,3 \%$ de adultos mayores con somnolencia diurna reportados por Durán et al. ${ }^{(18)}$ que indican el tomar una cena o comida después de las 21 horas y una edad mayor a 80 años como los principales factores relacionados con la somnolencia diurna en los participantes de su estudio. En cambio, en la presente investigación no se encontró asociación entre la edad de los participantes y la somnolencia diurna, ni se indagó sobre los hábitos alimentarios. Sin embargo, cabe destacar que las situaciones en las cuales las personas reconocen una mayor probabilidad de dormirse son estar sentado viendo la TV, o recostado a media tarde. Recordando que sólo un $32,18 \%$ de los participantes refería no tener una buena calidad de sueño, esto coincide con lo afirmado por la Guía de Práctica Clínica para el Manejo de Pacientes con Insomnio en Atención Primaria: muchos pacientes no perciben sus problemas de sueño hasta que se les pregunta específicamente sobre ellos $^{(17)}$, por ello será importante consultar sobre los problemas de sueño de los AM dentro del proceso de la valoración regular por parte de los profesionales de la salud. Del mismo modo, es muy importante incluir intervenciones multicomponentes (que consideren actividad física, estimulación cognitiva, higiene del sueño y alimentación) y que se puedan hacer con los AM en la comunidad para prevenir las alternaciones del sueño. Específicamente en lo que respecta a la actividad física, tanto ejercicios aeróbicos de baja a moderada intensidad, como los de flexibilidad y fuerza, por sí solos traen beneficios significativos a los parámetros del sueño, y deberán ser incluidos ${ }^{(22)}$.

Un $65,84 \%$ de los participantes percibía un alto nivel de apoyo social, siendo la dimensión "Apoyo afectivo" la mejor evaluada, mientras la que obtuvo la puntuación más baja fue "Apoyo instrumental". Es decir, las personas tienden a percibir un mayor apoyo relacionado con las demostraciones reales de amor, cariño o empatía, así como el tener con quién divertirse o pasar un buen rato, que aquel referente a la provisión de ayuda material, tangible, que pueda recibir el encuestado. 
Estos resultados son contrarios con lo observado por otros autores, como Revillaet al. ${ }^{(23)}$ quienes refieren que, a mayor edad, menor percepción de este apoyo social, así como una percepción más baja entre las mujeres. Jarrin et al. ${ }^{(15)}$, en cambio, destacan la relación entre vulnerabilidad económica e insomnio. Así también con lo descrito por Vivaldi que reporta que las personas AM con parejas (casados y convivientes) informan mayor percepción de apoyo social que las personas sin pareja (solteras, separadas y viudas) $)^{(11)}$.

Sí se encontró correlaciones significativas, si bien de pequeña magnitud, entre la severidad del insomnio y la somnolencia diurna, lo cual es esperable, pues un mal dormir se relaciona fisiológicamente con una menor capacidad de mantenerse alerta durante el día(15).

Más relevante resulta la asociación negativa encontrada entre la severidad del insomnio y el apoyo social percibido, tanto de forma global como en todas sus dimensiones. Es decir, un mayor apoyo social se relaciona con una menor prevalencia y severidad del insomnio, lo cual coincide con lo encontrado por Eshkoor et al. ${ }^{(14)}$, en AM con demencia, quienes sostienen que el aislamiento social aumenta las disrupciones del sueño; así como con el estudio de Viana et al. en AM brasileños ${ }^{(13)}$, quienes encontraron una menor percepción del apoyo social en las personas con más alteraciones del sueño, al igual que lo reportado por Matsumoto et al. ${ }^{(24)}$.

Otro antecedente que aporta al análisis es lo encontrado en un estudio realizado en el norte de Chile en 418 AM, y que tenía por propósito analizar cómo la estructura de la red (tamaño y frecuencia) y los elementos cualitativos y funcionales del apoyo (satisfacción y reciprocidad) interactúan y se relacionan con la salud de los AM, utilizando un modelo teórico basado en procesos que vinculan estructura y funciones del apoyo como elementos intrínsecamente relacionados, tratando de superar la dicotomía clásica entre ambos, realizando por medio de un análisis de ecuaciones estructurales. Ellos encontraron que las variables estructurales carecen en el modelo de un papel explicativo de la salud, entonces, el tamaño de la red aportaría el contexto. También, encontraron que las variables que tienen un papel protagónico para la salud en el caso de los AM son la satisfacción con los contactos en la red de apoyo y las medidas de los aspectos funcionales ${ }^{(25)}$. Ello podría explicar que un mayor apoyo social percibido, desde lo funcional, mejora la salud, y en este caso el Insomnio en los AM como problema de salud y síndrome geriátrico prevalente en este grupo etario.

También, se ha encontrado que los $A M$ que poseen un apoyo social percibido alto presentan mayores niveles de calidad de vida, es así como las personas que reciben un mayor apoyo social en términos de conversaciones telefónicas y visitas con amigos, familiares, vecinos y participación en actividades sociales, gozan de mejor salud ${ }^{(26)}$.

Según el modelo anterior, reviste especial atención el papel desempeñado por las distintas fuentes en la movilización de relaciones sociales satisfactorias que generen apoyo social eficaz como un proceso protector de la salud en la vejez ${ }^{(25)}$. En este contexto, es de destacar que en la actualidad, muchas intervenciones de AM en la comunidad están colocando el acento en la actividad física, estimulación cognitiva y autocuidado, de forma multicomponente. Sin embargo, los antecedentes de la presente investigación dan luces de la importancia psicosocial de estas intervenciones sociocomunitarias en los AM como razón o justificación de la misma intervención. Ya que el hecho de participar en este tipo de programas permite establecer redes de contacto extrafamiliar, evitar el aislamiento y la soledad, y favorecer aspectos de la salud mental de los AM, como tener un sentido en la vida, generar sentimientos de pertenencia e identidad ${ }^{(11)}$, y que también se traducirán en un buen dormir.

Por ello, también se recomienda la incorporación de instrumentos que evalúen del apoyo social percibido en los AM dentro de la batería de la Valoración Gerontológica integral en el contexto comunitario y de APS (como la escala MOS) dada su brevedad en tiempos de administración, sencillez, validez y aplicabilidad a estos contextos ${ }^{(27)}$, en particular en los programas gubernamentales y socio comunitarios, para pesquisar a los AM con bajo apoyo social percibido, que junto a otros problemas de salud y síndromes geriátricos, se beneficien de intervenciones más ajustadas con un enfoque 
biopsicosocial y de forma interdisciplinar para mejorar la salud, el bienestar subjetivo y la calidad de vida de estos AM.

En cuanto a la relación entre apoyo social y somnolencia diurna, sólo se encontró relación estadísticamente significativa entre la dimensión "Interacción social positiva" con una menor somnolencia diurna. Podría explicarse por el hecho de que una mayor disponibilidad para reunirse con otras personas para divertirse o pasarlo bien ayuda a los AM a mantenerse en estado de alerta con mayor facilidad.

Entre las fortalezas de esta investigación, se puede mencionar el haber logrado el acceso a una muestra estadísticamente representativa de la población AM residente en la comuna, la utilización de instrumentos validados y estandarizados, que presentaron una consistencia interna buena a excelente además de que la relación entre estas variables es un tema poco estudiado en la literatura revisada a la fecha, en especial en Latinoamérica, encontrándose pocos estudios referentes a las características del sueño en AM. Ello plantea la necesidad de profundizar las investigaciones en torno al apoyo social percibido, los trastornos del sueño y otros síndromes geriátricos de la esfera mental, el bienestar subjetivo y la calidad de vida, ello en el curso de vida de Ios AM. Del mismo modo, será interesante investigar en el futuro como los desastres naturales, las desestabilizaciones políticas, sociales y económicas de los países (situación que están viviendo varios países de la región), que limitan la participación de los AM en las intervenciones de la comunidad y APS, afectan su participación social, su apoyo social percibido, aumentan el estrés, y en consecuencia, puedan aumentar los problemas en la cantidad y calidad del sueño de este grupo poblacional.

Algunas posibles limitaciones del estudio son la gran disparidad entre la proporción de hombres y mujeres participantes y el alto porcentaje de personas con estudios superiores en la muestra. Además, por tratarse de un estudio transversal, no se puede hablar de causalidad en la relación entre las variables.

Los AM participantes del estudio que presentaron un alto nivel de apoyo social presentaron una menor prevalencia y severidad del insomnio. Por lo tanto, el diseño y aplicación de intervenciones que fomenten la construcción y fortalecimiento de redes de apoyo social para los AM, bajo el enfoque del Modelo de Atención Integral en Salud, sería beneficioso para lograr en los AM una mejor calidad de sueño, y con esto un mayor bienestar y funcionalidad.

\section{REFERENCIAS BIBLIOGRÁFICAS}

1. Poblete $F$, Glasinovic A, Sapag J, Barticevic N, Arenas A, Padilla O. Apoyo social y salud cardiovascular: adaptación de una escala de apoyo social en pacientes hipertensos y diabéticos en la Atención Primaria chilena. Aten Primaria. 2015; 47(8):523-31.

2. González F, Massad C, Lavanderos F. Estudio Nacional de la Dependencia en las Personas Mayores. Santiago: Servicio Nacional del Adulto Mayor SENAMA; 2009. p. 1-122.

3. Muñoz C, Rojas P, Marzuca G. Criterios de valoración geriátrica integral en adultos mayores con dependencia moderada y severa en Centros de Atención Primaria en Chile. Rev Med Chil. 2015; 143(5):612-8.

4. Ministerio de Salud de Chile. Encuesta Nacional de Salud ENS Chile 2009-2010 [Internet]. Santiago: Ministerio de Salud - MINSAL; 2011 [cited 2019 Nov 12]. p. 1-1064. Available from: https://www.minsal.cl/portal/url/item/b

cb03d7bc28b64dfe040010165012d23.p df

5. Ropper A, Samuels M, Klein J. Adams y Victor. Principios de neurología. 10th ed. Ciudad de México: McGraw-Hill; 2013.

6. Durán S, Mattar P, Bravo N, Moreno C, Reyes S. Asociación entre calidad de vida y cantidad de sueño en adultos mayores de la Región Metropolitana y Región de Valparaíso, Chile. Rev Med Chil. 2014; 142(11):1371-6.

7. Segrin C, Domschke T. Social support, Ioneliness, recuperative processes, and their direct and indirect effects on health. Health Commun. 2011; 26(3):221-32.

8. Cuadra A, Medina E, Salazar K. Relación de bienestar psicológico, apoyo social, estado de salud física y mental con calidad de vida en adultos mayores de la ciudad de Arica. Límite Rev Interdiscip Filos y Psicol. 2016; 11(5):56-67.

9. Fortmann A, Gallo L. Social support and nocturnal blood pressure dipping: A 
systematic review. Am J Hypertens. 2013; 26(3):302-10.

10. Waller K, Mortensen $\mathrm{E}$, Avlund K, Osler $M$, Fagerlund $B$, Lauritzen $M$, et al. Subjective sleep quality and daytime sleepiness in late midlife and their association with age-related changes in cognition. Sleep Med. 2016;17: 16573.

11. Vivaldi F, Barra E. Bienestar Psicológico, Apoyo Social Percibido y Percepción de Salud en Adultos Mayores. Ter Psicol. 2012; 30(2):23-9.

12. Glenn C, Enwerem N, Odeyemi $\mathrm{Y}$, Mehari A, Gillum R. Social Support and Sleep Symptoms in US Adults. J Clin Sleep Med. 2015; 11(8):957.

13. Da Costa $S$, Ceolim $M$, Neri A. Problemas de sueño y soporte social: estudio multicéntrico sobre fragilidad en ancianos Brasileños. Rev Lat Am Enfermagem. 2011; 19(4):920-7.

14. Eshkoor S, Hamid T, Nudin SS, Mun C. The effects of sleep quality, physical activity, and environmental quality on the risk of falls in dementia. Am J Alzheimers Dis Other Demen. 2013; 28(4):403-7.

15. Jarrin $D$, Chen I, Ivers $H$, Morin $C$. The role of vulnerability in stress-related insomnia, social support and coping styles on incidence and persistence of insomnia. J Sleep Res. $2014 ; 23(6): 681-8$.

16. Fernandez J, Rodriguez $A$, Vela $A$, Olavarrieta S, Calhoun S, Bixler E, et al. The Spanish version of the Insomnia Severity Index: A confirmatory factor analysis. Sleep Med. 2012; 13(2):20710.

17. Grupo de Trabajo de la Guía de Práctica Clínica para el Manejo de Pacientes con Insomnio en Atención Primaria. Guía de Práctica Clínica para el Manejo de Pacientes con Insomnio en Atención Primaria. Madrid: Unidad de Evaluación de Tecnologías Sanitarias. Agencia Laín Entralgo. Comunidad de Madrid; 2009. p. 5-170. Available from: https://portal.guiasalud.es/wpcontent/uploads/2018/12/GPC_465_Ins omnio_Lain_Entr_compl.pdf

18. Durán S, Sánchez H, Díaz V, Araya M. Factores asociados a la somnolencia diurna en adultos mayores chilenos. Rev Esp Geriatr Gerontol. 2015; 50(6):270-3.

19. Abusleme $M$, Contreras N, Garmendia A, Massad C. Indicadores
Sociodemográficos de las Personas Mayores a Nivel Territorial [Internet]. Santiago: Unidad de Estudios, División de Planificación y Desarrollo, Servicio Nacional del Adulto Mayor; 2013 [cited 2019 Nov 12]. p. 1-104. Available from:

http://www.senama.gob.cl/storage/doc s/Indicadores-Sociodemograficos-delas-Personas-Mayores-Nivel-Territorial2013.pdf

20. Galleguillos D. Inclusión social y calidad de vida en la vejez, Experiencias de personas mayores participantes en clubes de adultos mayores en la comuna de Talagante [Internet]. Universidad de Chile, Facultad de Ciencias Sociales, Departamento de Antropología; 2015 [cited 2019 Nov 12]. Available from: http://repositorio.uchile.cl/bitstream/ha ndle/2250/142331/memoria.pdf?sequen $\mathrm{ce}=1$ \&isAllowed $=\mathrm{y}$

21. Gabelle A, Gutierrez L-A, Jaussent I, Navucet S, Grasselli C, Bennys K, et al. Excessive Sleepiness and Longer Nighttime in Bed Increase the Risk of Cognitive Decline in Frail Elderly Subjects: The MAPT-Sleep Study. Front Aging Neurosci. 2017; 9:1-11.

22. Moreno $P$, Muñoz $C$, Pizarro $R$, Jiménez S. Efectos del ejercicio físico sobre la calidad del sueño, insomnio y somnolencia diurna en personas mayores. Revisión de la literatura. Rev Esp Geriatr Gerontol. 2019 (in press);

23. Crowley K. Sleep and Sleep Disorders in Older Adults. Neuropsychol Rev. 2011 Mar 12; 21(1):41-53.

24. Matsumoto $S$, Yamaoka $K$, Inoue $M$, Muto S. Social Ties May Play a Critical Role in Mitigating Sleep Difficulties in Disaster-Affected Communities: A Cross-Sectional Study in the Ishinomaki Area, Japan. Sleep. 2014;37(1):13745.

25. Gallardo L, Barrón A, Sánchez E, Arias A. Dimensiones estructurales $y$ funcionales del apoyo social y salud en personas mayores en Chile. Rev Mex Psicol. 2014; 31(2):187-97.

26. Alfonso L, Carballo D, Santos N. Calidad de vida y apoyo social percibido en adultos mayores. Rev Ciencias Médicas Pinar del Río. 2016; 20(1).

27. Ahumada R, Del Castillo L, Muñoz B, Moruno M. Validación del cuestionario MOS de apoyo social en Atención Primaria. Med Fam. 2005;6(1):10-8. 